\title{
Desarrollo de la economía creativa en Argentina: ejemplo para el caso colombiano
}

DOI: https://doi.org/10.21158/01208160.n0.2020.2753

Fecha de recepción:19 de mayo de 2020

Fecha de aprobación: 06 de noviembre de 2020

Fecha de publicación: 14 de diciembre de 2020
Hans David Falkenstein-Ávila ${ }^{1}$

Universitaria Agustiniana

hans.falkenstein@uniagustiniana.edu.co

Andrea Carolina Redondo-Méndez ${ }^{2}$

Universitaria Agustiniana

andrea.redondo@uniagustiniana.edu.co

Carlos Andrés Pinzón-Muñoz ${ }^{3}$

Universitaria Agustiniana

carlos.pinzonn@uniagustiniana.edu.co

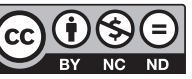

Cómo citar este artículo / To reference this article / Comment citer cet article / Para citar este artigo:

Falkenstein-Ávila, H. D.; Redondo-Méndez, A. C.; Pinzón-Muñoz, C. A. (2020). Desarrollo de la economía creativa en Argentina: ejemplo para el caso colombiano. Revista Escuela de Administración de Negocios, Edición especial 2020, 115 - 134. DOI: https://doi.org/10.21158/01208160.n0.2020.2753

\section{Resumen}

El objetivo del presente artículo es analizar la aplicabilidad de las estrategias de crecimiento del sector cultural argentino para el caso colombiano, a partir de las teorías del desarrollo de Stiglitz y la neoshumpeteriana de innovación, todo esto mediante tres variables de análisis: legislación, institucionalidad y cooperación. Argentina se convierte en un gran factor a tener en cuenta para desarrollar políticas, en términos de know-how, educación, capacitación e infraestructura, lo que permitiría analizar si Colombia puede lograr ventajas competitivas en el mercado internacional para el sector cultural. Se utilizó una metodología de tipo cualitativo y con enfoque documental, utilizando fuentes secundarias que permitieron la identificación de las estrategias y el análisis de las posibilidades de aplicación. Como principal resultado se identifica el proceso endógeno de crecimiento del sector cultural argentino, al vincular la diversidad institucional y normativa, así como la educación y la integración de la sociedad civil en la construcción de ciudades creativas, como lo es el caso de Buenos Aires. Para el caso colombiano, se evidencia la necesidad de estrategias que vayan más allá de lo institucional y lo normativo para apoyar al sector cultural, de modo que vinculen mayores presupuestos y mayor inclusión, para integrar a todos los artistas y creadores colombianos en la toma de decisiones y la creación de políticas.

Palabras clave: economía creativa; sector cultural; teorías del desarrollo Stiglitz; teoría neoshumpeteriana de innovación; políticas culturales.

\footnotetext{
${ }^{1}$ Estudiante de Negocios Internacionales - Universitaria Agustiniana. Miembro del semillero de investigación SINI. ORCID: https:// orcid.org/0000-0002-2076-5778

${ }^{2}$ Politóloga - Universidad Nacional de Colombia. Especialista en Docencia - Universidad Militar Nueva Granda. Magíster en Investigación Social Interdisciplinar - Universidad Distrital Francisco José de Caldas. ORCID https://orcid.org/0000-0001-92268626

${ }^{3}$ Profesional en Comercio Internacional - Fundación Universitaria Jorge Tadeo Lozano. Especialista en evaluación y desarrollo de proyectos - Universidad Nuestra Señora del Rosario. Magíster en Desarrollo sostenible y medio ambiente - Universidad de Manizales. ORCID: https://orcid.org/0000-0001-8376-0504
} 


\section{Development of the creative economy in Argentina: example for the Colombian case}

\section{Abstract}

The aim of this article is to analyze the applicability of the growth strategies for the Argentinean cultural sector to the Colombian case, based on Stiglitz's development theories and the neo-Shumpeterian theory of innovation, all through three variables of analysis: legislation, institutionality, and cooperation. Argentina has become a major factor to be taken into account when developing policies, in terms of know-how, education, training, and infrastructure, which would allow us to analyze whether Colombia can achieve competitive advantages in the international market for the cultural sector. We used a qualitative methodology with a documentary approach, including secondary sources that allowed the identification of strategies and the analysis of the possibilities for implementation. As the main result, we identified the endogenous process of growth of the Argentinean cultural sector, as it links institutional and regulatory diversity, as well as education, and integrates civil society in the construction of creative cities, as is the case of Buenos Aires. In the case of Colombia, there is a need for strategies that go beyond the institutional and normative to support the cultural sector, so that it is possible to link larger budgets and greater inclusion, in order to integrate all Colombian artists and creators in decision-making and policy making.

Keywords: creative economy; cultural sector; Stiglitz development theories; neo-Shumpeterian innovation theory; cultural policies.

\section{Desenvolvimento da economia criativa na Argentina: exemplo para o caso colombiano}

\section{Resumo}

O objetivo deste artigo é analisar a aplicabilidade das estratégias de crescimento do setor cultural argentino para o caso colombiano, com base nas teorias de desenvolvimento de Stiglitz e da neo-shumpeteriana da inovação, tudo isso por meio de três variáveis de análise: legislação, institucionalidade e cooperação. A Argentina passa a ser um grande fator a ser levado em consideração no desenvolvimento de políticas, em termos de know-how, educação, formação e infraestrutura, que permite analisar se a Colômbia pode obter vantagens competitivas no mercado internacional do setor cultural. Foi utilizada uma metodologia qualitativa com abordagem documental, utilizando fontes secundárias que permitiram a identificação de estratégias e a análise das possibilidades de aplicação. Como principal resultado, identificou-se o processo de crescimento endógeno do setor cultural argentino, ao articular a diversidade institucional e normativa, bem como a educação e a integração da sociedade civil na construção de cidades criativas, como é o caso de Buenos Aires. Para o caso colombiano, evidencia-se a necessidade de estratégias que vão além do institucional e do normativo para apoiar o setor cultural, de forma que vinculem orçamentos mais elevados e maior inclusão, para integrar todos os artistas e criadores colombianos na tomada de decisões e na criação de políticas.

Palavras-chave: economia criativa; setor cultural; teorias de desenvolvimento de Stiglitz; teoria neoshumpeteriana da inovação; políticas culturais. 


\section{Développement de l'économie créative en Argentine: un exemple pour la Colombie}

\section{Résumé}

L’objectif de cet article est d’analyser l'applicabilité des stratégies de croissance du secteur culturel argentin pour les transposer au cas colombien en se basant sur les théories du développement de Stiglitz et de l'innovation néoshumpétérienne au travers de trois variables d'analyse que sont la législation, l'institutionnalité et la coopération. L'Argentine est une source d'inspiration importante en terme d'élaboration de politiques, de savoir-faire, d'éducation, de formation et d'infrastructure, et l'analyse des facteurs clés de succés permettrait de savoir si la Colombie pourrait obtenir des avantages compétitifs sur le marché international du secteur culturel. Nous avons mis en place une méthodologie qualitative, d'approche documentaire, en utilisant des sources secondaires permettant l'identification de stratégies et l'analyse des champs d'application. Le principal résultat de cette investigation repose sur l'identification du processus de croissance endogène du secteur culturel argentin fusionnant la diversité institutionnelle et normative, l'éducation et l'intégration de la société civile pour la construction de villes créatives comme Buenos Aires. En ce qui concerne la Colombie, l'analyse montre qu'il est nécessaire de créer des stratégies de soutien au secteur culturel, allant au-delà du simple cadre institutionnel et normatif, pour allouer des budgets plus élevés et associer tous les artistes et créateurs aux prises de décisions et à l'élaboration des politiques culturelles.

Mots-clés: économie créative; secteur culturel; théories du développement de Stiglitz; théorie néoshumpétérienne de l'innovation; politiques culturelles. 


\section{Introducción}

$\mathrm{D}$ esde el siglo XX los Estados-nación han empezado a reconocer la unión y el trabajo conjunto entre cultura y comercio, dado que es un aspecto fundamental para el desarrollo de sus economías, ya que no solo se trata de una aseveración de aspectos identitarios resaltados, sino de la utilización de la cultura como catalizadora de emprendimientos, receptora de empleabilidad, de inclusión social y de recepción de inversión. Esto ha generado que el establecimiento de las industrias culturales y creativas se haya conglomerado bajo el concepto de economía creativa, según lo cual, en palabras de Hewsion, «es la configuración de las relaciones lo que le da al sistema sus características esenciales. Por lo tanto, es menos útil definir la economía creativa por lo que hace, que tratar de entender cómo está organizada» (2014).

La organización de la economía creativa vincula los rendimientos y las estructuras de las industrias culturales y creativas, y los gobiernos guían y establecen las rutas por las que estas logran su impulso. De acuerdo con la Conferencia de las Naciones Unidas sobre Comercio y Desarrollo (UNCTAD, 2018), el mercado global para bienes creativos se duplicó de USD 208 mil millones en el 2002 a USD 509 mil millones durante el periodo 2002-2015. En Latinoamérica, además del aumento de las cifras positivamente, se ha presentado un proceso de implementación de políticas de desarrollo en algunas de las economías más importantes de la región con el fin de motivar el progreso de la economía y contribuir así a los objetivos de desarrollo en la zona.

En el recorrido de los Estados en la economía creativa se han instaurado varios planes de inclusión social y de desarrollo con el fin de cultivar los mejores resultados. De esta manera, según el Programa de las Naciones Unidas para el Desarrollo, países como Argentina han centrado su atención en la «creación de políticas que hacen parte de dicha economía para las industrias digitales, audiovisuales y de diseño» (PNUD y Unesco, 2014). Actualmente, estos son los sectores más desarrollados en ese país, y esto ha iniciado un modelo más reciente que incluye nuevas tecnologías. Por otra parte, en países como Colombia, de manera más habitual se ha mantenido una visión más «tradicional como lo es el arte y el patrimonio» (PNUD y Unesco, 2014).

El objetivo de la investigación fue analizar la aplicabilidad de las estrategias de crecimiento del sector cultural argentino para el caso colombiano a partir de las teorías del desarrollo de Stiglitz y la neoshumpeteriana de innovación. El artículo presenta inicialmente una serie de definiciones de la economía creativa y de las industrias culturales y creativas, así como su estructuración en los dos países de estudio. Luego establece los principales logros del Estado argentino en el desarrollo de la economía creativa, y a continuación describir el papel de Buenos Aires como fuerza, impulso y ejemplo de ciudad creativa, es decir, como ejemplo de desarrollo y crecimiento para el país. En seguida se plantean las estrategias, divididas en legislación, institucionalidad y cooperación, desde acuerdos internacionales, para, finalmente, y utilizando las teorías de desarrollo y de organización de Joseph Stiglitz y la teoría neoshumpeteriana, realizar un análisis de las estrategias argentinas identificadas, aplicadas al caso colombiano, con lo que se reconoce su utilidad.

De esta manera, se trata de la descripción y el análisis de cada uno de los aportes, teniendo en cuenta que,

el sector cultural está provisto de una variedad de oportunidades de crecimiento social y económico, dentro de estándares de desarrollo e inclusión, debido a que pone en el centro de su realización una serie de productos culturales y creativos que generan ingresos significativos para cualquier Estado. (Redondo-Méndez, 2019, p. 42) 
Es de especial relevancia entender el desarrollo del sector cultural como aporte latinoamericano, en cuanto factor determinante de crecimiento y protagonista del crecimiento económico.

De esta manera, Argentina, como uno de los países líderes en el sector creativo, podrá aportar pautas y políticas a países como Colombia para que la estructura de esta economía sea más efectiva. Sin embargo, no se debe olvidar que el crecimiento del sector cultural depende de la relevancia que se le dé a la economía creativa, pero en conjunto; es decir, a la totalidad de actores primarios y secundarios que confluyen y que se desarrollan dentro del llamado ecosistema creativo. No basta con estrategias adaptadas de modelos exitosos si no existe un sistema inclusivo de artistas y creadores, más el apoyo de un gobierno consiente del aporte y la valía de un sector en crecimiento.

\section{Metodología}

$\mathrm{C}_{\text {es de tipo cualitativo, ya que reconoce los }}^{1 \text { enfoque de la investigación, en primer lugar, }}$ planteamientos,

en lugar de que la claridad sobre las preguntas de investigación e hipótesis preceda a la recolección y el análisis de los datos - como en la mayoría de los estudios cuantitativos-, los estudios cualitativos pueden desarrollar preguntas e hipótesis antes, durante o después de la recolección y el análisis de los datos. Con frecuencia, estas actividades sirven, primero, para descubrir cuáles son las preguntas de investigación más importantes, y después, para refinarlas y responderlas. (Hernández, Fernández y Baptista, 2004, p. 7)

De esta manera, se analizaron documentos aportados por entidades gubernamentales internacionales tales como el Banco Interamericano de Desarrollo (BID) o dependencias de la Organización de las Naciones Unidas (ONU), así como por nacionales de la Argentina y Colombia. De igual forma, se maneja información de entidades privadas con el fin de establecer los niveles participativos que tienen en la economía creativa de cada país a indagar.

También se planteó como una investigación exploratoria, debido a que realiza un acercamiento al sector cultural de dos países e identifica fortalezas en sus estrategias y la situación coyuntural de cada uno. Finalmente, es de tipo descriptivo, en la medida en que pretende evidenciar las características de la economía creativa en Argentina más importantes y sus acciones desarrolladas o implementadas frente al desarrollo del sector cultural, con el propósito de analizarlas en el caso colombiano. 


\section{Resultados}

\subsection{Economía creativa}

El conglomerado del sector cultural, a nivel global, ha manifestado una transformación relevante dentro de los Estados actuales, al ser reconocido como garante de crecimiento económico y en su calidad de recepto de procesos innovadores desde y para la sociedad civil. Como parte fundamental de este sector se encuentra la creatividad, entendida desde diversas vertientes, pero reconocida acá como un elemento inherente al ser humano, es decir, a la manera de un proceso de desarrollo personal que se define como una capacidad, o como,

el conjunto de aptitudes vinculadas a la personalidad del ser humano que le permiten, a partir de una información previa, y mediante una serie de procesos internos - cognitivos-, en los cuales se transforma dicha información, la solución de problemas con originalidad y eficacia. (Hernández, 1999, p. 67)

Ahora bien, esa creatividad, esa capacidad o esas aptitudes, han incursionado en esferas múltiples de la sociedad estableciendo su importancia y su necesaria aplicación. Tal es el caso de la economía, la cual abre una esfera nueva de aplicación y se combina con esta, de manera que da origen a la llamada economía creativa. En palabras de Jhon Howkins (2005), su creador:

Por «economía creativa» se entienden las
operaciones que generan los productos creativos -
resultantes-. Cada operación puede comportar dos
valores complementarios: el valor de la propiedad
intelectual intangible y el valor de la plataforma física
— de haberla-. En algunos sectores, como el de los
programas informáticos digitales, es mayor el valor
de la propiedad intelectual. En otros, como las artes,
el valor unitario del objeto físico es más elevado.

Por esto, en el marco de la economía creativa se logra identificar las actividades, las industrias y los subsectores que la componen, sin olvidar que cada sector - tradicional o no- que incluya el elemento creatividad dentro de sus procesos también será considerado como parte de la economía creativa o aportante de esta. Así, entonces, quienes componen directamente la economía creativa son las industrias culturales y creativas y sus propios componentes, entendiendo que son estas las que la mueven, la desarrollan y le generan resultados.

3.1.1 Industrias culturales y creativas en Colombia y en Argentina.

Para el Gobierno colombiano, este tipo de industrias $\ll$ se caracterizan por realizar actividades coordinadas entre sí, para que las ideas se transformen en bienes y servicios culturales y/o creativos. El valor de estos bienes está determinado, en muchos casos, por el contenido de propiedad intelectual que albergan» (Mincultura, 2018, pár. 1). Esto es, se trata del conglomerado de actividades que ofrecen bienes y servicios culturales, con alto valor agregado desde el uso de la creatividad. Para el caso argentino, se trata de actividades vinculadas con la creación, la producción y la comercialización de bienes y servicios culturales que, además, establecen sinergias con sectores tradicionales y aportan de manera significativa al PIB del país y a la generación de empleo.

Sin embargo, y sin decir que son definiciones contrarias, ambos países coinciden en la definición de la Organización de Naciones Unidas para la Educación, la Ciencia y la Cultura (Unesco), según la cual las industrias culturales son «aquellos sectores de actividad organizada que tienen como objeto principal la producción o la reproducción, la promoción, la difusión y/o comercialización de bienes, servicios y actividades de contenido cultural, artístico o patrimonial» (Unesco, 2009, p. 21).

A partir de lo anterior, el complemento teórico de la investigación utiliza la teoría de los círculos concéntricos de David Throsby (2008), quien define las industrias culturales y creativas a partir de su ubicación y el uso o la creatividad de su aplicabilidad. 
Así, definir las industrias culturales y creativas supone el entendimiento de su modelo, el cual demuestra que el origen de las ideas creativas está en las artes creativas, esto es, música, literatura y artes visuales, es decir, se forman a partir del sonido, el texto y la imagen; así mismo, a medida que se van alejando, lo cultural disminuye de manera progresiva y aparece lo creativo, que se difunde hacia afuera a través de unos círculos concéntricos. Esto genera cuatro círculos concéntricos:

- Literatura, música, artes escénicas, artes visuales -industrias creativas centrales-
- Cine, museos, bibliotecas, galerías, fotografía otras industrias culturales centrales-. Industrias cuyos productos son exclusivamente culturales $y$ destinados a la reproducción y difusión en masa, es decir, las llamadas industrias culturales.

- Patrimonio, medios impresos, edición, televisión, radio, videojuegos —industrias culturales más amplias-.

- Publicidad, diseño, moda y arquitectura diferentes sectores productivos-. La cultura se convierte en una entrada «creativa» en la producción de bienes no culturales.

Figura 1. Modelo de círculos concéntricos de las industrias culturales y creativas

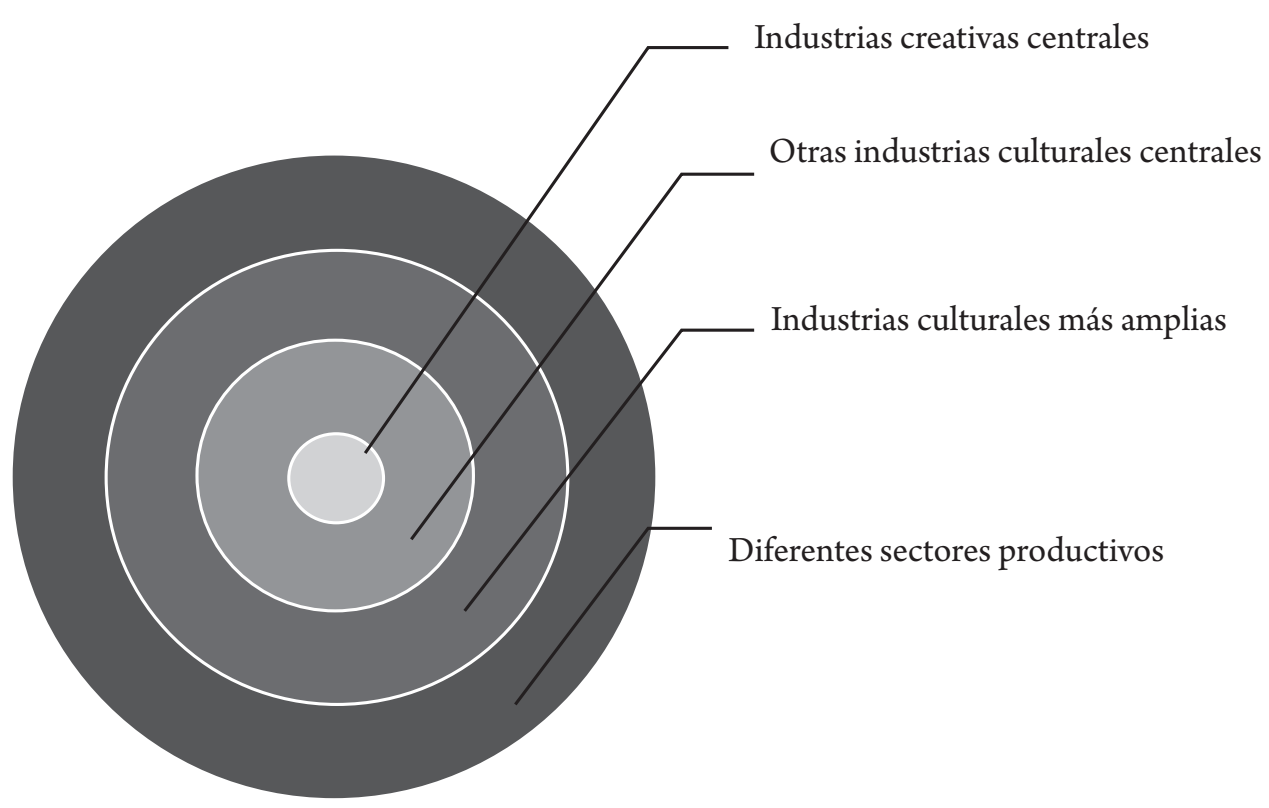

Fuente. Throsby, 2008. 
A partir de la figura 1 es posible identificar el papel de las industrias culturales y creativas como engranajes de mayor desarrollo del sector cultural, en la medida en que el círculo interior se establece como el generador de la creación y como la base para los demás círculos; en el segundo círculo se encuentran las industrias que están en ámbitos de creatividad; en el tercer círculo las industrias que facilitan la reproducción, distribución y consumo masivo de contenidos o producciones culturales; y en el cuarto círculo se presentan las industrias creativas de carácter aplicado.

En Colombia, la economía creativa se divide en tres categorías, generadas a partir de las divisiones mundialmente aceptadas y mencionadas, de modo que se crean categorías sectoriales para su reconocimiento, valoración y transformación. La primera categoría es artes y patrimonio, incluye turismo y patrimonio cultural material e inmaterial, así como la educación en artes, cultura y economía creativa; la segunda categoría es industrias culturales, e incluye las industrias editorial, fonográfica y audiovisual; la tercera es creaciones funcionales, nuevos medios y software de contenidos, e incluye medios digitales y software de contenidos, diseño y publicidad.

En Argentina también se tienen tres grandes grupos definidos para evaluar y analizar el sector cultural; el primero, compuesto por audiovisual, publicidad y contenido digital; el segundo por editorial, diseño, música y artes escénicas; y el tercero patrimonio material, formación cultural y artes plásticas y visuales.

\subsection{Estrategias en la economía creativa de Argentina}

Actualmente la economía creativa se ha convertido en un referente mundial, útil para realizar mediciones acerca de diferentes «expresiones culturales» que puedan existir y que se están generando. Es funcional en el propósito de ser una plataforma inclusiva que toma en cuenta los desarrollos tecnológicos, por lo que Latinoamérica se ha caracterizado por ser una región muy importante en el momento de efectuar aportes a las cifras resultantes de la economía creativa; es una región que ha mostrado crecimiento y avance no solo en la producción o generación de bienes y servicios culturales, sino también en la regulación, la organización y el apoyo.

Otra característica importante de Latinoamérica es la posibilidad de catalogar la región como una zona que empieza a trabajar mancomunadamente para desarrollar cada vez más la economía creativa. Con esto, dentro de los estudios que realizan el Banco Interamericano de Desarrollo (BID) o dependencias de la Organización de las Naciones Unidas (ONU), entre otros, se evidenció que,

la conciencia sobre la importancia socioeconómica de la economía creativa se expande a través del tiempo en esta región del mundo, a su vez es interesante observar que las políticas son tomadas en cuentas más por las autoridades municipales que a un nivel federal. (UNCTAD, 2010, p. 92)

Argentina, por su parte, se ha convertido en un referente del desarrollo de políticas, instituciones, alianzas público-privadas y otras actividades importantes que le han servido para llevar a ese país al estatus de éxito que tiene actualmente, compartido con Brasil y Chile. Mucho de esto tiene que ver con su sistema de mapeo georreferencial, el cual ha permitido reconocer las actividades del sector, la ubicación geográfica y los retos y victorias que manifiesta.

Así mismo, gracias a esto ha sido posible evidenciar a través de los análisis en los que ha participado Argentina, entre los que se cuentan investigaciones lideradas por ellos mismos, algunos de los estudios más importantes. Estos han sido «Economía creativa. Informe 2010», «Economía creativa. Informe 2014», «EY cultural times 2015. Nosotros y los otros 2008», liderado por Argentina y Venezuela», o «Capital intangible en las cadenas globales de valor 2017», entre otros. Esto ayuda a identificar que Argentina, en cuanto a su economía creativa, se tiene en cuenta en los debates y estudios globales por su experticia, su mantenimiento y la proyección que tiene para este sector. 


\subsubsection{Principales logros.}

Argentina se ha reconocido como un generador de nuevos negocios a nivel regional, lo que a su vez permite que los empleados y el aumento de la balanza comercial —en cuanto a las exportacionesmejoren de manera significativa. Consecuentemente, ese país ha logrado implementar procesos y políticas que le han servido como factor crucial al desarrollar su economía creativa, la cual se ha caracterizado por ser un $\ll$ sector [que] tiene una gran cantidad de mano de obra y se basa en las artes y las artesanías» (PUND y Unesco, 2014). Sin dejar atrás las tradicionales, como lo son las artes y el patrimonio, también se «han instaurado políticas para las industrias digitales, audiovisuales y de diseño» (2014).

Económicamente la creatividad a lo largo del último siglo en Argentina ha obtenido resultados importantes en el desarrollo de su actividad de economía creativa. En este caso, el Instituto Nacional de Estadística y Censos de Argentina ayuda a generar un contexto, de manera que se pueden hallar resultados constantes, como, por ejemplo:

La tasa media de crecimiento del sector cultural fue de 7,8 \% durante el periodo 2003-2011, y su cuota del PIB pasó de 2,47 \% en 2004 a 3,83 \% en 2011, habiendo crecido por séptimo año consecutivo. La industria del libro destacó especialmente, produciendo 31.691 títulos y 118.700 .987 de ejemplares, una cifra récord. Dos iniciativas federales, pero implementadas a nivel local. La contribución del diseño al PIB nacional y al comercio exterior de Argentina es indiscutible. Entre 2005 y 2007, el sector creció un $12 \%$ anual y generó ganancias de exportación de $\$ 700$ millones. En cuanto al atuendo, las industrias del cuero y el calzado generaron en 2007 una facturación de alrededor de \$ 2.6 mil millones, representando el $3 \%$ del total economía y el 2,5 por ciento del empleo total en la capital argentina. (PNUD y Unesco, 2014)

Además de las cifras que el PNUD y la Unesco agregan, el Instituto Nacional de Estadística y Censos (Indec) de Argentina, en conjunto con el Ministerio de Cultura de ese país, elaboran una medición acerca del impacto económico de la economía creativa sobre su PIB, también denominado «el valor agregado» (VAB); es la suma de los valores monetarios de los bienes y los servicios producidos en un determinado periodo (Jara, 1 de noviembre de 2015). En la figura 2 se evidencia el crecimiento que ha tenido el impacto en el PIB o VAB de Argentina entre los años 2004 y 2011.

Figura 2. VAB Argentina 2004-2011

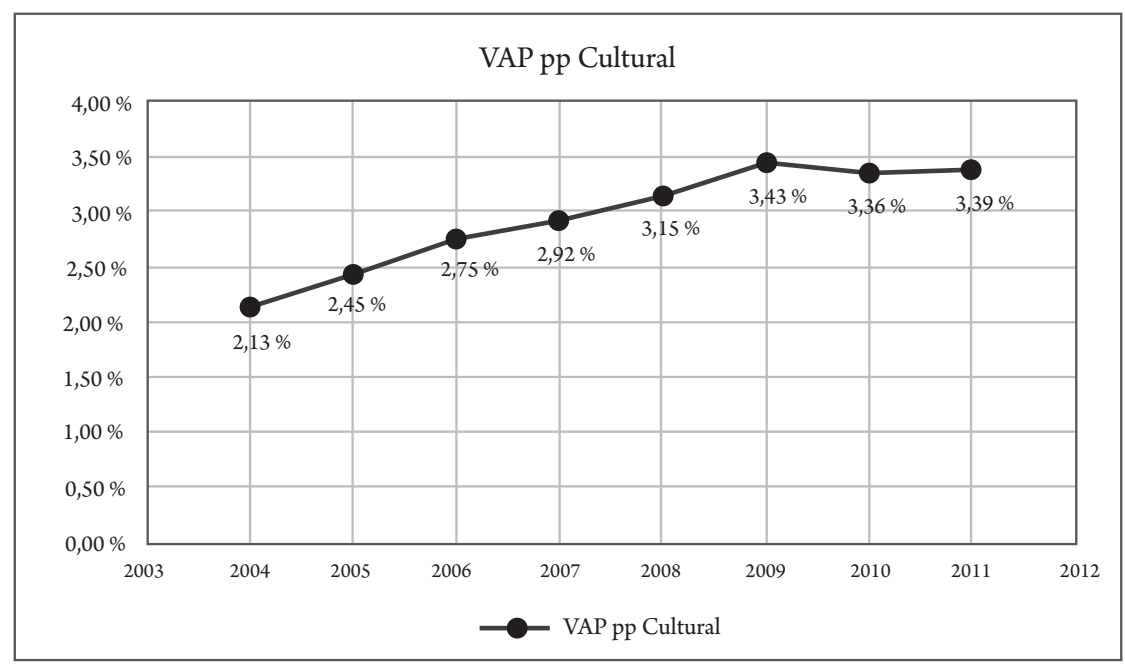

Fuente. Elaboración propia con base en Secretaría de Cultura de la Presidencia de la Nación, 2012. 
Dentro del rango tomado en cuenta en el estudio, el 2009 es el año que más crecimiento presentó. Si se tienen en cuenta las variaciones que ha venido presentando la economía argentina, el sector económico ha tenido un crecimiento importante, de modo que representa el 3,39 \% de esa economía. Así mismo, varias secretarías dependientes del Gobierno de Argentina, entre ellas la Secretaría de Política Económica y Planificación del Desarrollo, la Subsecretaría de Planificación Económica, la Dirección Nacional de Planificación Regional y la Dirección Nacional de Planificación Sectorial elaboran un informe anual en el que explican:

En 2014, la cadena dio cuenta del 3,70 \% del valor agregado de la economía y su crecimiento en 2015 se estima en 0,5\%, ligeramente por debajo del promedio de la economía. Si se considera la contribución de cada subsector, es posible observar que el audiovisual es el más importante - 39,1\%-y su participación es creciente entre el 2010 y 2014. En segundo lugar, se encuentra publicidad - 13,3\%y en tercer lugar, libros y publicaciones - 10,7 \%-; ambos perdieron participación entre en el periodo 2010-2014. El rápido crecimiento de los contenidos digitales en los últimos años le permitió alcanzar una participación del 6,3\%. Las actividades deportivas y de esparcimiento contribuyen con el 0,97 \% del PIB. (Gobierno de Argentina, 2016)

En cuanto al comercio exterior de Argentina en materia de la economía creativa, se ha presentado un crecimiento y una diversificación importante si se tiene en cuenta que, según el informe elaborado por encargados de los ministerios de Cultura de Argentina y Venezuela, coincidieron en lo siguiente:

Al analizar la información para el caso argentino, puede observarse una marcada diferencia: mientras tan solo dos países explican casi la totalidad de las importaciones —Brasil y México, con el 59,5\% y el $38 \%$ respectivamente-, para el caso de las exportaciones hay una notoria diversificación regional en relación a la participación de los países de destino. Al respecto, es importante señalar que la incidencia de México y Brasil es notoriamente menor para las exportaciones: México solo explica el 11,7\%, mientras que Brasil el $29 \%$. Tres países limítrofes -Uruguay (14\%), Chile (11 \%) y Paraguay (6\%) - explican poco menos de una tercera parte de las exportaciones culturales argentinas en 2006.

(Calcagno y Lerman, 2008)

Sin embargo, en el último informe dado a conocer por el Gobierno de Argentina y participantes de algunas secretarías, se comprueba dicha diversificación, lo que convierte a otros países en aliados estratégicos para la economía creativa de ese país. En este sentido, el informe indica:

Esta cadena presenta un alto potencial para la expansión y generación de puestos de trabajo. Los programas de televisión y las películas están cada vez más orientados a la exportación a nivel mundial. Argentina está en el ranking global de los cinco mayores exportadores de formatos y guiones, junto con Estados Unidos, Gran Bretaña, España y Colombia. Los formatos latinoamericanos tienen como destinos a Rusia, Francia y Norteamérica, entre otros. (Gobierno de Argentina, 2016)

Gracias a la diversificación de entidades encargadas de detallar las diferentes variables de la economía de Argentina, ha sido posible detallar información hasta incluso el 2017, en la cual se refleja que las exportaciones del sector están lideradas por el subsector de los libros y las publicaciones, así como por las artes plásticas y visuales (véase la Figura 3). 
Figura 3. Exportaciones de cultura-Argentina 2017

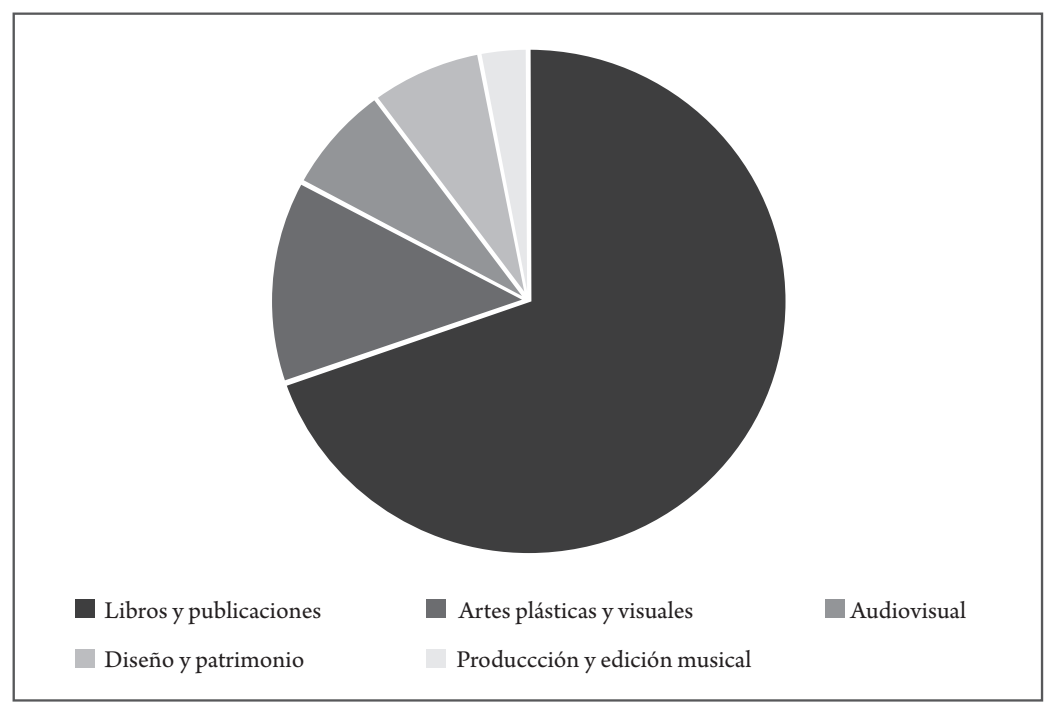

Fuente. Elaboración propia con base en Ministerio de Cultura, 2018.

3.2.2 Buenos Aires: la fuerza de la economía creativa.

Gracias al trabajo que se ha realizado en la capital de Argentina, Buenos Aires se ha convertido en una de las ciudades representativas a nivel mundial en lo que a la economía creativa respecta; en este caso, gracias al trabajo mancomunado público-privado, tal como lo indica el informe de la Unesco y el PNUD (2014): «Buenos Aires se ha convertido en un punto de referencia global por su estrategia de industrias creativas. Su Observatorio de Industrias Creativas produce estadísticas, indicadores y estudios de forma regular». Esto ha permitido no solo a la ciudad, sino al país desarrollar sobre la marcha las políticas necesarias para mejorar cada vez más, de modo que con esto «la mayoría de sus estudios se llevan a cabo para entidades tanto públicas como privadas y se centran en la intersección entre cultura y tecnología» (PNUD y Unesco, 2014).

De esta manera, Buenos Aires no solo ha logrado consolidarse como una de las más importantes en la economía creativa, sino también en materia de resultados cuantitativos: «Según el Anuario 2011. Industrias Creativas de la Ciudad de Buenos Aires, el valor añadido generado por las industrias creativas ha ido creciendo no menos de $5 \%$ al año, arrojando un total de $73,1 \%$ entre 2004 y 2010 a precios constantes». Así mismo, de manera general, «el PIB de la ciudad aumentó en un 49,2\% durante el mismo periodo» (PNUD y Unesco, 2014).

Parte de su éxito como ciudad creativa se debe al trabajo institucional desarrollado por el Gobierno argentino, en la medida en que reúne expertos en cada área y les permite desarrollar estrategias basadas siempre en el mapeo y la construcción de datos. Algunos ejemplos de esto se ven reflejados en el Centro Metropolitano de Diseño de Buenos Aires, una institución pública que apoya y promueve industrias creativas relacionadas con el diseño, así como busca fomentar la competitividad en mercados nacionales e internacionales. También la UNCTAD ha estado colaborando con el Gobierno, en particular con el Ministerio de Desarrollo Económico y el secretario de Cultura, a fin de promover aún más la economía creativa en el país, como parte de una iniciativa gubernamental dirigida a fomentar el avance de la economía creativa. Con esto,

otras ciudades en el país también están activas promoviendo sus economías creativas. Rosario ha incluido en el calendario de la ciudad el «Rosario Active», un evento anual eso es parte de una 
estrategia para estimular la creatividad y creatividad emprendimiento para acelerar el desarrollo urbano. (Falkenstein-Avila, 2020, p. 120)

También en el Observatorio de Industrias Culturales de la Provincia de Buenos Aires, el cual, a su vez, es dependiente del Instituto Cultural a cargo de Jorge Telerman. El Observatorio de Industrias Creativas (OIC) es una unidad de estudios conformada por un equipo interdisciplinario y dedicada a la obtención, elaboración y difusión de información cuantitativa y cualitativa sobre las industrias creativas (IC) locales. El principal objetivo del OIC es contribuir a la conformación de un sistema de información sobre las IC que sirva a la toma de decisiones tanto por parte de la gestión pública como por parte de los actores que intervienen en la producción cultural y creativa (Buenos Aires Ciudad, s. f.). En este sentido, la ciudad de Buenos Aires tiene algunas ventajas comparativas vinculadas especialmente al buen nivel creativo, técnico y profesional en muchas de las industrias creativas, lo cual se suma, en algunos sectores, a una larga tradición empresarial local.

En uno de los informes presentados por la ciudad sobre las economías e industrias creativas es posible identificar con claridad fortalezas que pueden indicar el avance que tiene esta ciudad. En primera instancia, la capital argentina cuenta con una amplia red de escuelas dedicadas a la enseñanza y la formación profesional de diferentes profesiones relacionadas con las industrias creativas. Así mismo, la ciudad hace referencia a factores que generan distinción regional de excelencia en términos de formación y de producción de las industrias de contenidos con las que cuenta la ciudad, pues allí el recurso humano y la creatividad se constituyen en ingredientes distintivos, lo que generaría otro factor importante a tener en cuenta en el éxito que la ciudad presenta.

De igual forma, una de las estrategias fundamentales ha sido el Mercado de Industrias Culturales Argentinas (MICA), una iniciativa intersectorial que está conjuntamente organizado por las siguientes instituciones nacionales: la Secretaría de Cultura, el Ministerio de Industria, el Ministerio de Relaciones
Exteriores, Comercio Internacional y Culto, el Ministerio de Trabajo, Empleo y Seguridad Social, el Ministerio de Turismo y el Ministerio de Planificación Federal, Inversión Pública y Servicios.

Así, el MICA organiza un espacio único en el que se reúne una serie de actividades, y apoya al mismo tiempo las diferentes cadenas de la industria cultural. Con el objetivo de promover negocios, fomentar el intercambio de información y la creación de redes profesionales entre las distintas regiones de Argentina, y proyectar al resto del mundo la calidad, competitividad y diversidad de las industrias culturales nacionales, el MICA se centra en las artes escénicas, la producción audiovisual, el diseño, la industria editorial, la música y los videojuegos. Creado en el 2011 e inicialmente organizado en Buenos Aires, la primera edición nacional del MICA tuvo lugar en el 2012 y, a continuación, fue descentralizada para su operación en seis regiones. Los resultados han sido impresionantes, dado que ha alcanzado a 2200 empresas y ha organizado 7400 rondas de negocios (PNUD y Unesco, 2014).

\subsubsection{Reconocimiento de estrategias.}

Las estrategias reconocidas para el caso argentino se agruparon en institucionalidad, normatividad y cooperación internacional, y enmarcan la totalidad de programas y ayudas existentes para el desarrollo de la economía creativa del país. A continuación, se mencionan algunos de los espacios generados a partir de las estrategias y los espacios seleccionados (véase la Tabla 1).

El Sistema de Información Cultural de la Argentina (SINCA) es un organismo que hace parte del Ministerio de Cultura argentino y su función es generar, sistematizar y difundir información de las actividades culturales del país; cuenta con más de 20 instituciones asociadas para el levantamiento de información, su análisis y su presentación. Justamente, este organismo pudo determinar que el sector creativo lo componen un poco más de 400000 empleaos formales, según su último censo, quienes integran sectores como los siguientes: 
La industria gráfica, los servicios de logística, transporte y comunicación o la producción de eventos. A la vez, hay muchas otras actividades donde la identidad, la creatividad, el conocimiento y la información son insumos diferenciales que agregan valor a sus productos. Es el caso del turismo, la publicidad, los medios digitales interactivos o la gastronomía, entre otros. (Gribnico, 2017)

Otra de las estrategias es el trabajo colectivo, en el cual el sector público, a través de un trabajo mancomunado, busca el desarrollo de la economía creativa Argentina y, «a partir de la creación de la Subsecretaría de Economía Creativa en la órbita de la Secretaría de Cultura y Creatividad del Ministerio de Cultura, Argentina cuenta hoy con un plan estratégico orientado al desarrollo de este universo productivo» (Gribnico, 2017). Así mismo, dentro del trabajo en paralelo del sector público y privado,

[la] Red de Ciudades Creativas, conformada por más de cincuenta ciudades argentinas que ven a la creatividad como una herramienta estratégica para su progreso a partir de los valores locales. Conectamos - en cada territorio - a la comunidad creativa, para visualizar lo que damos en llamar Ecosistema Creativo, un mapeo exhaustivo de todos los que conforman esta cadena de valor - artistas, creativos, empresarios, instituciones públicas y privadas-. El principal objetivo es fortalecer en cada ciudad los ecosistemas creativos locales, a través de la promoción sectorial de las industrias creativas y su impacto en la economía local, impulsando el trabajo colaborativo entre todos los actores que la conforman. (Gribnico, 2017)

De esta manera, el trabajo conjunto de las ciudades se vuelve importante; sin embargo, existe un factor que debe tenerse muy en cuenta a fin de que la estructura planteada funcione: la educación como la forma más efectiva para obtener resultados sin dejar de lado el financiamiento y la innovación, lo cuales,

se originan tanto en el propio Ministerio de Cultura y sus organismos descentralizados como en otras áreas del Estado nacional, como son la Agencia Argentina de Inversiones y Comercio Internacional, el Ministerio de Producción, el Ministerio de Ciencia, Tecnología e Innovación Productiva, el Ministerio de Turismo, el Ministerio de Trabajo o el Ministerio de Relaciones Exteriores y Culto. (Gribnico, 2017)

Al integrar las entidades nombradas se llega a una de las actividades más importantes del comercio creativo de la Argentina que se hayan podido implementar antes, pues allí se encuentra la ronda de negociaciones del Mercado de Industrias Creativas de Argentina (MICA), el cual ha tenido un impacto tan relevante en Latinoamérica y algunos países europeos que, incluso, tuvo sede en Bogotá para su desarrollo. En este caso, como indica Ana Carla Fonseca en el informe de PNUD:

MICA organiza un espacio único en el que se reúnen una serie de actividades, apoyando al mismo tiempo las diferentes cadenas de la industria cultural. Con el objetivo de promover negocios, fomentar el intercambio de información y la creación de redes profesionales entre las distintas regiones de Argentina, así como proyectar al resto del mundo la calidad, competitividad y diversidad de las industrias culturales nacionales, el MICA se centra en las artes escénicas, la producción audiovisual, el diseño, la industria editorial, la música y los videojuegos. Creado en 2011 e inicialmente organizado en Buenos Aires, la primera edición nacional del MICA tuvo lugar en 2012 y a continuación fue descentralizada para su operación en seis regiones. Los resultados han sido impresionantes, habiendo alcanzado a 2.200 empresas y organizado 7.400 rondas de negocios. (PNUD y Unesco, 2014)

Otro factor clave del desarrollo de la economía creativa de ese país ha sido su transición hacia una economía creativa más ligada o complementada con la tecnología; aunque no deja de lado la economía creativa tradicional. Así pues,

se están preparando iniciativas similares como parte de una estrategia para desarrollar su impacto a nivel internacional. El Distrito de la Tecnología ya tiene alrededor de 110 empresas creativas y de información tecnológica operando en lo que hasta ahora había sido un área ignorada de la ciudad. (PNUD y Unesco, 2014) 
En el marco del trabajo en conjunto de los sectores privados y públicos, también se han destacado nuevas iniciativas que han empezado a tomar relevancia, aunque similares a MICA, en busca de fuente de recursos para otros creativos:

Tabla 1. Estrategias de Argentina en la economía creativa
[El] Distrito de Diseño, así como el Distrito de las Artes, el cual se encuentra en desarrollo y tiene el objetivo de promover la inversión en arte y fortalecer la posición de Buenos Aires como ciudad cultural. El proyecto está orientado a compañías e individuos relacionados con las artes visuales, la música, los libros y las artes escénicas, y ofrece una serie de incentivos fiscales. (PNUD y Unesco, 2014)

\begin{tabular}{|c|c|c|}
\hline Factor & & Ítem \\
\hline $\begin{array}{l}\text { Legislación argentina } \\
\text { vigente }\end{array}$ & $\begin{array}{r}\text { i. } \\
\text { ii. } \\
\text { iii. } \\
\text { iv. } \\
\text { v. } \\
\text { vi. } \\
\text { vii. } \\
\text { viii. } \\
\text { x. } \\
\text { xi. } \\
\text { xii. } \\
\text { xiii. } \\
\text { xiv. } \\
\text { xv. } \\
\text { xvi. } \\
\text { xvii. } \\
\text { xviii. } \\
\text { xix. } \\
\text { xx. } \\
\text {. }\end{array}$ & $\begin{array}{l}\text { Decreto-1117/2018-10-12-2018. } \\
\text { Decreto-802/2018-05-09-2018. } \\
\text { Decreto de Necesidad y Urgencia-801/2018-05-09-2018. } \\
\text { Decreto de Necesidad y Urgencia-575/2018-22-06-2018. } \\
\text { Decreto de Necesidad y Urgencia-746/2017-26-09-2017. } \\
\text { Decreto de Necesidad y Urgencia-513/2017-17-07-2017. } \\
\text { Decreto de Necesidad y Urgencia-2/2017-03-01-2017. } \\
\text { Ministerio de Ambiente y Desarrollo Sustentable-Decreto De Necesidad y Urgencia-223/2016-20-01-2016. } \\
\text { Decreto De Necesidad y Urgencia-13/2015-11-12-2015. } \\
\text { Ley-22520-12-03-1992. } \\
\text { Presidencia de la Nación-Decreto-180/2019-11-03-2019. } \\
\text { Administración Pública Nacional-Decreto-105/2019-01-02-2019 } \\
\text { Administración Pública Nacional-Decreto-48/2019-14-01-2019. } \\
\text { Jefatura de Gabinete de Ministros-Decreto-1169/2018-26-12-2018. } \\
\text { Administración Pública Nacional-Decreto-1119/2018-11-12-2018. } \\
\text { Administración Pública Nacional-Decreto-958/2018-26-10-2018. } \\
\text { Administración Pública Nacional-Decreto-585/2018-26-06-2018. } \\
\text { Administración Pública Nacional-Decreto-428/2018-10-05-2018. } \\
\text { Ministerio de Ciencia, Tecnología e Innovación Productiva-Decreto-350/2018-23-04-2018. } \\
\text { Administración Pública Nacional-Decreto-174/2018-05-03-2018. } \\
\text { Otros } 360 \text { decretos y sanciones legales. }\end{array}$ \\
\hline Institucionalidad & $\begin{array}{r}\text { i. } \\
\text { ii. } \\
\text { iii. } \\
\text { iv. } \\
\mathrm{v} . \\
\text { vi. } \\
\text { vii. } \\
\text { viii. } \\
\text { ix. } \\
\text { x. } \\
\text { xi. } \\
\text { xii. } \\
\text { xiii. } \\
\text { xiv. } \\
\text { xv. } \\
\text { xvi. } \\
\text { xvii. } \\
\text { xviii. } \\
\text { xix. } \\
\text { xx. } \\
\text { xxi. } \\
\text { xxii. } \\
\text { xxiii. } \\
\text { xxiv. } \\
\text { xxv. } \\
\text { xxvi. } \\
\text { xxvii. } \\
\text { xxviii. } \\
\text { xxix. } \\
\text { xxx. }\end{array}$ & $\begin{array}{l}\text { Ministerio de Cultura. } \\
\text { Subsecretaría de Economía Creativa. } \\
\text { Secretaría de Cultura y Creatividad. } \\
\text { Sistema de Información Cultural de la Argentina. } \\
\text { Convenio Andrés Bello. } \\
\text { Red de Ciudades Creativas. } \\
\text { Agencia Argentina de Inversiones y Comercio Internacional. } \\
\text { Ministerio de Producción. } \\
\text { Ministerio de Ciencia, Tecnología e Innovación Productiva. } \\
\text { Ministerio de Turismo. } \\
\text { Ministerio de Trabajo. } \\
\text { Ministerio de Relaciones Exteriores y Culto. } \\
\text { Mercado de Industrias Creativas de Argentina (MICA). } \\
\text { Secretaría De Patrimonio Cultural. } \\
\text { Biblioteca Nacional Dr. Mariano Moreno. } \\
\text { Cinemateca y Archivo de la Imagen Nacional. } \\
\text { Fondo Nacional de las Artes. } \\
\text { Ballet Nacional. } \\
\text { Inst. Nac. «J. D. Pérón» de Est. e Inv. Hist., Soc. y Polít. } \\
\text { Instituto Nacional de Teatro. } \\
\text { Teatro Nacional Cervantes. } \\
\text { Comisión Nacional de Monumentos, de Lugares y Bienes Históricos. } \\
\text { Instituto Nacional de Investigaciones Históricas Eva Perón. } \\
\text { Instituto Nacional de Investigaciones Históricas Juan Manuel De Rosas. } \\
\text { Museo Nacional de Bellas Artes. } \\
\text { Instituto Nacional Sanmartiniano. } \\
\text { Instituto Nacional Belgraniano. } \\
\text { Instituto Nacional Browniano. } \\
\text { Instituto Nacional Newbriano. } \\
\text { Instituto Nacional Yrigoyeniano. }\end{array}$ \\
\hline
\end{tabular}




\begin{tabular}{|c|c|c|}
\hline Institucionalidad & $\begin{array}{r}\text { xxxi. } \\
\text { xxxii. } \\
\text { xxxiii. } \\
\text { xxxiv. } \\
\text { xxxv. } \\
\text { xxxvi. }\end{array}$ & $\begin{array}{l}\text { Cn. Protectora de Bibliotecas Populares. } \\
\text { Casa Creativa del Sur. } \\
\text { Secretaría de Coord. de Gestión Cultural. } \\
\text { Secretaría de Gobierno de Cultura. } \\
\text { Instituto Nacional de la Musica (Inamu). } \\
\text { Inst. Nac. de Cine y Artes Audiovisuales (Incaa). }\end{array}$ \\
\hline $\begin{array}{l}\text { Acuerdos } \\
\text { internacionales }\end{array}$ & \multicolumn{2}{|r|}{$\begin{array}{l}\text { En la actualidad existen más de } 130 \text { proyectos de cooperación técnica en diferentes regiones del mundo. Los ejes de } \\
\text { trabajo que los países demandan a la hora de asociarse con Argentina son aquellos en los cuales nuestro país muestra } \\
\text { un valor agregado: agroindustria, innovación tecnológica productiva/ciencia y tecnología, industrias creativas, medio } \\
\text { ambiente, salud y derechos humanos. Así mismo, y en términos de distribución geográfica, América Latina ha sido } \\
\text { la región más beneficiada. En los últimos años, África y Asia han cobrado un dinamismo particular, llevándonos a } \\
\text { aumentar la presencia en Sudáfrica, Mozambique, Tailandia, China y Singapur, entre otros (Cancillería de Argentina, s. } \\
\text { f.). }\end{array}$} \\
\hline
\end{tabular}

Fuente. Elaboración propia.

Es válido resaltar el trabajo que se ha desarrollado en conjunto con los sectores privados y públicos con miras a que factores como la innovación, las tecnologías y, en mayor medida, la educación, se desarrollen y permitan así que Argentina sea uno de los epicentros de desarrollo de la economía creativa a escala global, de modo que se resalte su participación en Latinoamérica.

\section{Discusión}

\begin{abstract}
A través de la identificación de algunas de las principales estrategias que ha implementado, desarrollado y que tiene actualmente Argentina, se establecen los factores importantes para constituir objetivos y posibilidades dirigidos a efectuar una planificación estratégica con el fin de incrementar el desarrollo de la economía creativa en Colombia. Gracias a la tipificación se logra identificar una ruta alineada con los supuestos de Stiglitz y Shumpeter,
\end{abstract}

encaminada hacia un desarrollo venidero, así como a estar en capacidad de establecer acciones sólidas frente al mercado cultural actual.

A continuación, se presenta el análisis de las estrategias identificadas -mediante los tres escenarios propuestos-, a través de las tablas 2, 3 y 4, teniendo como referente las teorías del desarrollo y la organización de Stiglitz y la innovación neoshumpeteriana. 
Tabla 2. Neoclásica: Stiglitz —instituciones—

\begin{tabular}{|c|c|c|}
\hline Aporte de Stiglitz & Argentina & Colombia \\
\hline Instituciones & $\begin{array}{l}\text { A través del breve recorrido hecho por la } \\
\text { composición de las entidades públicas o mixtas, e } \\
\text { incluso algunas privadas que trabajan en conjunto } \\
\text { en el propósito de llevar a una realidad la economía } \\
\text { creativa en Argentina, se identificaron algunas } \\
\text { que están exclusivamente dirigidas a la economía } \\
\text { creativa. En ese sentido, algunas también dependen } \\
\text { de una gran superficie como lo es la Subsecretaría } \\
\text { de Economía Creativa, adscrita al Ministerio de } \\
\text { Cultura de Argentina. } \\
\text { Una característica a resaltar es el trabajo } \\
\text { mancomunado que llevan a cabo las } \\
\text { administraciones de cada ciudad argentina, las } \\
\text { cuales, en conjunto, conforman la Red de Ciudades } \\
\text { Creativas, de modo que sirve como plataforma } \\
\text { para trabajar en las necesidades de manera más } \\
\text { específica, es decir, un trabajo más detallado en lo } \\
\text { que a la economía creativa respecta. }\end{array}$ & $\begin{array}{l}\text { En Colombia el desarrollo de la economía creativa } \\
\text { se ha establecido durante los últimos dos años } \\
\text {-2018 a 2020- a partir del Plan Nacional de } \\
\text { Desarrollo 2018-2022, ylas 7i, en las que se reúnen, } \\
\text { a través de ejes estratégicos, las instituciones } \\
\text { que entrarán a participar del desarrollo de este } \\
\text { sector; a saber, Mincultura, Mincomercio, DANE, } \\
\text { Bancoldex, Innpulsa, Mineducación, SENA, } \\
\text { MinTic y Procolombia. }\end{array}$ \\
\hline
\end{tabular}

Fuente. Elaboración propia.

La tarea de desarrollar instituciones quizás sea una de las actividades que más trabajo costaría no solo en cuanto a lo financiero, también en cuanto al diseño organizacional para que se dé un trabajo eficiente. Este sería uno de los eslabones más difíciles de construir. Sin embargo, el trabajo que ha realizado el Gobierno de Argentina con miras a desarrollar las instituciones ha acarreado una gran labor e inversiones, además de conocimiento económico. Para el caso colombiano, hasta la implantación del gobierno actual se empezaron a identificar instituciones que, si bien ya existían, tienen un espacio para el sector cultural. La diferencia o el punto crítico radica en que, mientras en Argentina existen instituciones exclusivas para la cultura, en Colombia se deben compartir con otros sectores y con más funciones.

Tabla 3. Neoclásica: Stiglitz —organización—

\begin{tabular}{|c|c|c|}
\hline Aporte de Stiglitz & Argentina & Colombia \\
\hline \multirow{3}{*}{ Organización } & \multirow{3}{*}{$\begin{array}{l}\text { El Sinca y el MICA, en cuanto factores que } \\
\text { proporcionan al sistema de economía creativa } \\
\text { argentino un valor agregado e innovador por el } \\
\text { apalancamiento de nuevos negocios; ya sea de } \\
\text { inversión nacional o extranjera y el mantenimiento } \\
\text { de la información actualizada de todo lo referente a la } \\
\text { creatividad; brindan un punto de partida significativo } \\
\text { para fortalecer o madurar el sector. } \\
\text { Con respecto al conjunto de normas que incluyan } \\
\text { mecanismos para el mejoramiento, implementación } \\
\text { o desarrollo mismo de la economía creativa se puede } \\
\text { tener en cuenta en este factor. }\end{array}$} & $\begin{array}{l}\text { Gracias a la Cuenta Satélite de Cultura del DANE } \\
\text { y el Ministerio de Cultura ha sido posible hacer un } \\
\text { mapeo del flujo económico y demás asociados a los } \\
\text { tipos de patrimonio en Colombia. }\end{array}$ \\
\hline & & $\begin{array}{l}\text { Cabe resaltar la participación de entidades privadas } \\
\text { como Invest in Bogotá, la cual ha brindado un } \\
\text { acercamiento al mundo de los negocios a la } \\
\text { economía creativa en Bogotá. }\end{array}$ \\
\hline & & $\begin{array}{l}\text { Además de ser fundamentales para la constitución } \\
\text { de las entidades públicas y de carácter mixto con el } \\
\text { fin de atender la economía creativa en Colombia, } \\
\text { se han impulsado algunas mejoras a los estamentos } \\
\text { que rigen hoy en día. }\end{array}$ \\
\hline
\end{tabular}

Fuente. Elaboración propia. 
En este caso, es importante resaltar el interés de algunas entidades en Colombia en realizar acercamientos con el fin de hacer negocios de manera nacional o internacional con economía creativa; sin embargo, puede que la tarea resulte compleja en el momento de ejecutar, no por falta de inversionistas o capital, sino por sistemas integrados que brinden la información necesaria para la toma de decisiones de la viabilidad de los negocios. No obstante, Bogotá, específicamente, ha participado en el MICA como un invitado, mercado en el que las industrias creativas de Argentina siguen llevando la delantera, ya que son los proveedores; en este sentido, la búsqueda va en el desarrollo de la economía creativa de Colombia.

Sin embargo, si se habla de las cuentas satélite de cultura de ambos países, la diferencia es evidente. Mientras Argentina cuenta con mapeo cultural, consumo cultural, mapeo georreferencial y datos de cada uno de los sectores y subsectores, Colombia no posee ni la caracterización completa del sector cultural en todo el país y apenas ha empezado una recogida de datos y un análisis de los beneficios a través de las participaciones de artistas y creadores en mercados internacionales y el reconocimiento cultural e identitario de algunas de sus prácticas. Un elemento también resaltado por Stiglitz en cuanto al desarrollo es la participación del capital humano como ficha clave para que el proceso de mejoramiento del sistema sea en principio realidad.

Se deberá contar con las personas que compondrán ese grupo de creativos emprendedores, así como quienes van a ayudar a la construcción de una economía creativa solidificada y más inclusiva. Para que esto sea posible, será necesario tener programas de capacitación y educativos que les permitan a las personas que componen el grupo conocer las ventajas de la economía creativa, como lo son la inclusión social, el desarrollo y el crecimiento económico, entre otras. Esto en el caso colombiano no se contempla de manera organizada o diferenciada, sino que se maneja sin sinergias entre la educación profesional y la no formal.

Tabla 4. Neoclásica: Stiglitz — desarrollo—

\begin{tabular}{|c|c|c|}
\hline Aporte de Stiglitz & Argentina & Colombia \\
\hline Desarrollo & $\begin{array}{l}\text { Una economía creativa atípica, es decir, partiendo } \\
\text { con implementaciones tecnológicas para que } \\
\text { industrias específicas - como el caso de la } \\
\text { cinematográfica- crezcan a través de acuerdos } \\
\text { de cooperación internacional con entidades } \\
\text { multilaterales y empresas privadas e incentivos } \\
\text { económicos, de manera que puede realizarse la } \\
\text { inversión bien sea de manera fiscal, o bien en el } \\
\text { desarrollo de software, entre otros. }\end{array}$ & $\begin{array}{l}\text { El desarrollo de software ha ganado terreno de } \\
\text { forma significativa en Colombia; sin embargo, } \\
\text { hasta donde fue posible evidenciar, no hay una } \\
\text { estructura que respalde el desarrollo de manera } \\
\text { amplificada o masiva de este sector creativo. }\end{array}$ \\
\hline
\end{tabular}

Fuente. Elaboración propia.

Con el fin de implementar un desarrollo que permita a la economía creativa de Colombia progresar de manera significativa, habrá que tomar como base las ejecuciones que Argentina realizó en su momento, tales como efectuar las mejoras que llevó a cabo con su modelo, e instaurar así una economía creativa con más innovaciones, sin dejar atrás el sector patrimonial ya avanzado. Ahora bien, desde otros sectores como el desarrollo de aplicaciones, de software, mercadeo y demás que le sirvan de apalancamiento para generar un comercio interno y externo más diversificado.
Con este propósito será necesario generar un aporte presupuestal mayor para el sector cultural, debido a que, si bien es una de las estrategias del gobierno actual, no se tiene destinada una cantidad suficiente al logro de estos objetivos.

Colombia tiene una ventaja frente a otros competidores internacionales en el ámbito de la innovación. En este sentido, como se mencionó en el informe de Banco Interamericano de Desarrollo, el país contó con una gran participación, sobre todo en los proyectos de desarrollo de software, lo que se 
convierte en el principal indicio de que el país tiene un avanzado capital humano para implementar y sujetarse a nuevos cambios. De acuerdo con Schumpeter, se aporta el proceso innovador como un elemento de vital trascendencia para que el desarrollo se pueda realizar, y en el caso de la economía creativa se vuelve un proceso imprescindible en su cadena de valor.

Aunque Schumpeter relata la mejor manera para lograr un impacto significativo en el sistema tradicional conformado por mejoramientos tecnológicos y sociales, es importante mencionar que Argentina está un paso adelante en este sentido, ya que ha implementado mejores modelos de educación, capacitación y formas de lograr facultar a sus habitantes para contar con los conocimientos necesarios, bien sea para emprender o bien escoger especializarse en alguno de los temas tratados.
Así mismo, el trabajo conjunto que deben realizar las empresas para generar un bienestar social hace que el posicionamiento de las industrias culturales cada día sea mejor frente a la región latinoamericana, con lo cual es necesario que las instituciones, como lo nombra Stiglitz, trabajen a fin de brindar las pautas principales. Schumpeter en este caso aborda las instituciones como una necesidad de fomento de una política monetaria mejorada. En el caso argentino se ha visto cómo Buenos Aires, por medio de varios estamentos, entre ellos las leyes de mecenazgos, permite que ciudadanos obtengan el respaldo económico de instituciones privados o públicas, de manera que los procesos innovadores se ven respaldados; esto supone una facilidad para que las personas escojan la economía creativa como su principal fuente de recursos.

\section{Conclusiones}

$\mathrm{L}$ os resultados de esta investigación muestran la posición superior que tiene Argentina frente al desarrollo de la economía creativa. Esto se demuestra no solo con su organización interna, sino con su posicionamiento y el apoyo gubernamental al sector cultural, que tiene mayor conocimiento en cuanto a su composición, mejor funcionamiento de sus instituciones y mayor aprovechamiento de sus recursos legislativos enfocados en la cultura.

Argentina demostró la existencia de un sistema sólido y replicable, ya que no muestra signos de dificultad más allá de una organización y asignación de tareas específicas a través de toda la cadena de valor. Así, reconoce cada uno de los protagonistas del ecosistema creativo de su país y son claras las funciones de cada uno. Algunos de los resultados que vale la pena resaltar es la diversificación, pero como un trabajo en conjunto de las instituciones; ejemplo de esto es la Red de Ciudades Creativas o la internacionalización de los negocios creativos como el MICA lo hace, de modo que en este caso son factores que robustecen la organización de esa economía, al ser espacios propicios para la transformación y la creación cultural.

Otro de los componentes que podría ser una de las fortalezas más importantes para el avance argentino es la capacidad de mantener la información de la industria y la economía creativa de forma suficiente, de modo que permita a los negocios prosperar y se genere atractivo para nuevos inversores tanto nacionales como internacionales; es decir, mantener un sector organizado genera derramamientos positivos o externalidades como la inversión extranjera, al evidenciar el crecimiento y el trabajo del sector.

Igualmente, se debe resaltar la educación, ámbito en el que Argentina ha entendido que se necesita una población mejor capacitada para lograr resultados y avances significativos, y que no se trata solo de educación artística o educación empresarial, sino la convergencia de ambas y la síntesis de conocimiento y reconocimiento de habilidades diferentes. 
Por su parte, Colombia ha mostrado que la economía creativa es un factor fuerte, importante y ampliamente desarrollable. Ejemplo de esto es el reconocimiento de entidades multilaterales en lo que respecta al trabajo de Colombia en materia de creatividad con la creación de una nueva legislación y programas de fomento para el sector cultural, así como reconocimientos mundiales de ciudades emblemáticas como Bogotá o Medellín.

Sin embargo, es imperativo empezar a migrar de una economía creativa, focalizada en el desarrollo del sector patrimonial — tradicional—, a una economía creativa diversificada con un amplio espectro en las tendencias mundiales como lo es el desarrollo tecnológico y digital, sin que se deje de lado el patrimonio como sector creativo, ya que gracias a esto Colombia se encuentra posicionada en la región.

Esto será posible únicamente con el apoyo y la participación activa de todos los actores del mapa cultural colombiano, a saber, los artistas y creadores, el Gobierno nacional, los canales de comunicación entre cliente y consumidor, las asociaciones empresariales, las instituciones de financiación y la población civil. De esta manera, el sector se podrá beneficiar al mejorar los alcances, e incluso implementar convenios o tratados cooperativos internacionales con un plan o una ruta de trabajo que incluya la tecnología como factor clave a mejorar.

Al comparar los países se hace evidente el alcance que tienen en diferentes factores cada uno de ellos. Se refleja que Colombia sí debería implementar y desarrollar un modelo mejorado para el auspicio de las economías creativas, no solo por la posibilidad de mejorar de forma sistemática las instituciones, la educación o culturizar a la ciudadanía, sino por el poder que tiene en inclusión social, desarrollo económico con bajos niveles de inversión y la facilidad de llevarse a los mercados de manera nacional e internacional. Con esto, la desigualdad social y la pobreza serían factores que se enfrentarían, así como el nacimiento de nuevas oportunidades laborales.
Colombia mostró que tiene los mismos factores que tiene Argentina en el ámbito económico cultural; sin embargo, necesitará ir más allá de implantar nuevas leyes con beneficios y planificación, pues se necesitan leyes que fortalezcan y lleven al acto las grandes oportunidades que tiene la economía creativa, pero además que la totalidad de artistas puedan acceder a estas y no se queden como regulaciones en el papel.

Finalmente, y como lecciones importantes, se debe mencionar la herramienta que le ha permitido a Argentina ocupar los primeros lugares en desarrollo de la economía creativa. Esta ha sido, inicialmente, el enfoque en concentrar el trabajo en grupo, lo que hace que cada ciudad vele por el desarrollo local de la economía creativa y procure obtener los mejores resultados. De igual forma, el papel trascendental que ha jugado la capital para la movilización de negocios, al contar con información clara y sólida, así como incentivos y subsidios que les permite a los emprendedores generar ideas de desarrollo. Esto no significa que Colombia no tenga algún tipo de incentivo, lo que significa es que se podrían estructurar de una manera tal que las personas tengan más acceso a las fuentes de ayuda.

También es importante destacar el proceso endógeno que ha desarrollado Argentina en el mejoramiento de la educación, la culturización de la población y los proyectos de mejoramiento continuo de las herramientas al alcance. Por ejemplo, la tecnología, le ha permitido desarrollar sectores y potencializar habilidades que reconocen la conformación de la economía creativa y el valor de cada creador, lo que incluye soportar e integrar a cada uno a los programas que posee. Así, Colombia tendrá que materializar y llevar a cabo acciones tangibles para lograr un posicionamiento completo, al menos en la región; acciones que no solo son de forma, no solo son nombres de instituciones o de programas, sino de acercamiento y conocimiento de toda una población que aporta al desarrollo del país desde sus propios talentos, su creatividad y sus habilidades. 


\section{Referencias}

Calcagno, N.; Lerman, G. (2008). Nosotros y los otros. El comercio exterior de bienes culturales en América del Sur. Indicadores Culturales/Argentina 2008, 69-76. Recuperado de https://bit.ly/3418OgK

Cancillería de Argentina. (s. f.). Cooperación internacional. Recuperado de https://www.cancilleria.gob.ar/es/politicaexterior/cooperacion-internacional

Falkenstein-Avila, H. D. (2020). Economía creativa: Cooperación Colombia - Argentina. En R. F. Díaz-Vásquez, Instituciones, gobierno y gestión pública. Investigación en Relaciones Internacionales. Tomo 2. (113-129). Bogotá: Centro de Estudios Sociedad y Espacio.

Gobierno de Argentina. (2016). Informes de cadenas de valor. Recuperado de https://bit.ly/37QUZ5Y

Gribnico, A. (7 de agosto de 2017). La economía creativa Argentina. Pressreader. Recuperado de https://bit. ly/3qMSDgU

Hernández, C. (1999). Manual de creatividad publicitaria. Madrid: Síntesis.

Hernández, R.; Fernández, C.; Baptista, P. (2004). Metodología de la investigación. México D. F.: McGraw-Hill.

Hewison, R. (2014). Cultural capital: the rise and fall of creative Britain. Londres: Verso Books.

Howkins, J. (2005). El motor de la creatividad en la economía creativa: entrevista a John Howkins. (D. Ghelfi, entrevistador)

Jara, L. (1 de noviembre de 2015). Acceso estadístico, actividad económica, indicadores. Observatorio Económico Social UNR. Recuperado de https://bit.ly/3gwVF4k

Mincultura. (2018). ABC de la economía naranja. Gobierno de Colombia. Recuperado de https://bit.ly/39W597X

Ministerio de Cultura de Argentina. (2018). Ministerio de Cultura de la Nación. Recuperado de https://bit.ly/344AEJk
PNUD y Unesco (Programa de las Naciones Unidas para el Desarrollo); Unesco. (2014). Informe sobre la economía creativa. Ampliar los cauces de desarrollo local. Recuperado de https://bit.ly/37RtYiP

Redondo-Méndez, A. C. (2018). Industrias culturales latinoamericanas y su aporte al desarrollo social. En. J. Hernández-Acosta; A. C. Redondo-Méndez; O. OspinaMartínez (Eds.) Industrias culturales y economía creativa en Latinoamérica. Desarrollo económico y social en la región. (2370). Bogotá: Editorial Uniagustiniana. DOI: https://doi. org/10.28970/9789585498143.1

Secretaría de Cultura de la Presidencia de la Nación. (2012). Cuenta Satélite de Cultura en la Argentina. Aspectos metodológicos para su construcción. Buenos Aires: Secretaría de Cultura de la Presidencia de la Nación, Indec. Recuperado de https://bit. ly/3nkjfnD

Throsby, D. (2008). The economics of cultural policy. Cambridge: Cambridge University Press. DOI: https://doi.org/10.1017/ CBO9780511845253

UNCTAD. (2010). Creative economy programme. Recuperado de https://bit.ly/3qLknTe

UNCTAD. (2018). Informe dobre las inversiones en el mundo. La inversión y las nuevas políticas industriales. Mensajes clave $y$ panorama general. Ginebra, Suiza: Naciones Unidas. Recuperado de https://bit.ly/3oG0tHj 